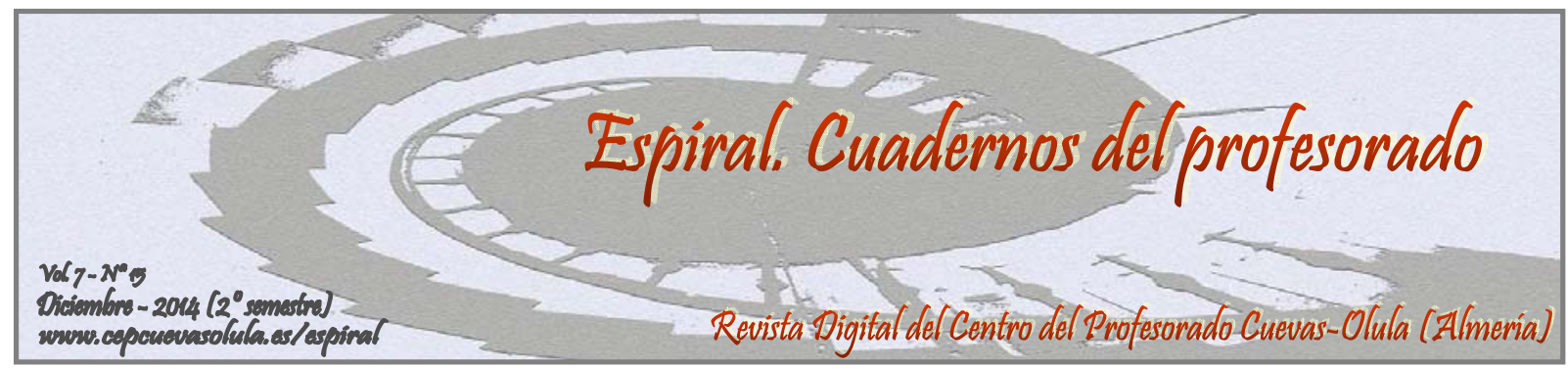

\title{
NUEVAS SALIDAS PROFESIONALES DE LOS GRADUADOS EN EDUCACIÓN FÍSICA Y CIENCIAS DEL DEPORTE: EL DEPORTE DE ORIENTACIÓN
}

\section{NEW PROFESSIONAL OUTPUTS FOR GRADUATES IN PHYSICAL EDUCATION AND SPORTS SCIENCE: ORIENTEERING}

\section{Antonio Baena-Extremera ${ }^{(1)}$, Daniel Jara Cortina ${ }^{(1)}$, Patricio Palao ${ }^{(1)}$, Humberto Luis de Deus Inàcio ${ }^{(2)}$ y Pedro J. Ruiz Montero ${ }^{(3)}$}

(1) Facultad de Ciencias del Deporte, Universidad de Murcia, España

(2) Facultad de Educación Física. Universidad Federal de Goias, Brasil

(3) Facultad de Ciencias de la Actividad Física y del Deporte, Universidad de Granada, España

RESUMEN: Las competiciones de raids de aventura están en aumento en los últimos años, existiendo ligas autonómicas y una liga nacional destacable. El objetivo del presente artículo es conocer la importancia que tiene la formación en orientación en pruebas de raids de aventura, y con ello, entender que la formación en este deporte puede ser una salida profesional futura. La muestra fue de 272 competidores de élite dentro de la Liga Española de Raids de Aventura, utilizando como instrumento un cuestionario autoadministrado validado previamente. Entre los resultados destaca que los raiders que practican orientación, son por lo general federados, llegando a ser campeones en algún tipo de carrera, sobre todo autonómica, nacional e internacional. Por esto, la formación en orientación en estas competiciones es muy importante.

Palabras clave: carreras de aventura, deporte de aventura, Educación Física.

ABSTRACT: Competitions adventure raids are on the rise in recent years, existing regional leagues and outstanding national league. The aim of this paper is to know the importance of training in counseling testing adventure races, and thus understand that training in this sport can be a future career path. The sample consisted of 272 elite competitors in the LERA, using a validated selfadministered questionnaire instrument previously. It finds that the raiders who practice guidance are usually federal, becoming champions in some kind of racers, particularly regional, national and international. Therefore, training in orientation in these competitions is very important.

Key words: adventure racing, adventure sport, physical education.

Baena-Extremera, A., Jara Cortina, D., Palao, P., de Deus Inàcio, H. L., y Ruiz Montero, P.J. (2014). Nuevas salidas profesionales de los graduados en Educación Física y ciencias del deporte: el deporte de orientación. Espiral. Cuadernos del Profesorado, 7(15), 49-54. Disponible en: http://www.cepcuevasolula.es/espiral. 


\section{1.- INTRODUCCIÓN}

Las actividades físicas en el medio natural (AFMN), son un contenido recogido a nivel curricular en la Educación Física en Secundaria, y que se trabaja también, en la etapa de primaria. Dentro de estos contenidos, y si seguimos los currículum escolares (Real Decreto 1513/2006 y Real Decreto 1631/2006), vemos que la orientación es uno de los más trabajados dentro del bloque de contenidos de AFMN.

Pero no solo a nivel escolar, la orientación está siendo un contenido en auge a lo largo de todo el mundo, publicándose numerosos trabajos tanto desde un punto de vista didáctico, como deportivo, de gestión, etc. Por ejemplo, a nivel deportivo, encontramos algunos trabajos como el de BaenaExtremera y Rebollo (2006), Baena-Extremera y Rebollo (2009), Schneider, Butryn, Furst, Masucci (2007), entre otros. En el grupo de artículos de didáctica, podemos destacar el trabajo de BaenaExtremera y Granero-Gallegos (2011), Casado (2010), Gómez (2003), Hellín y Giménez (1995), Kim (2010), López (2012), entre otros. Y así, podríamos destacar artículos de todos los ámbitos, relacionados con el deporte de orientación.

En el caso de las salidas profesionales de los graduados en Educación Física y Ciencias del Deporte, unificando tanto el entrenamiento, como la enseñanza, como la recreación, encontramos una salida nueva que es la de Técnico Deportivo en Orientación, enfocado a la enseñanza, el entrenamiento y la competición en orientación. Esto es importante, porque cada día son más numerosas las pruebas de trail, de raids y de combinadas de aventura donde se utiliza la orientación como vehículo central. Y creemos, que el futuro profesional, debería aprovechar esta oportunidad para buscar y crear un espacio de trabajo, como experto que es.

Para ratificar esta idea, el objetivo de esta investigación es presentar algunos datos obtenidos en relación al tipo de competidor que corre en los Raids de Aventura más importantes de España, demostrando que la formación en orientación, es importante para estos sujetos y para obtener éxitos en estas pruebas. Por ello, es necesario destacar esta futura salida profesional entre los universitarios.

\section{2.- MÉTODO}

\section{Población y muestra}

La población objeto de este estudio, la constituyen los participantes de la Liga Española de Raids de Aventura (2004-2005), la cual fue de 272 sujetos.

\section{Diseño y material}

Dadas las características propias de estas competiciones (riesgo de algunas pruebas, lugares de celebración, distancias y carga de trabajo físico,...), y puesto que no es posible tomar muestras durante la competición, se optó por trabajar con un diseño no experimental, descriptivo y seccional, utilizando un cuestionario al inicio de cada prueba.

\section{Procedimiento y análisis de datos}

El trabajo de campo se ha realizado a lo largo de las competiciones celebradas dentro de la LERA 2005-2006. Dentro de cada competición, la organización de cada prueba citaba a los competidores el día antes para realizar el penúltimo briefing, donde se aprovechaba para proporcionar el cuestionario de forma autoadministrada, que iba siendo rellenado equipo por equipo.

Para el análisis de los datos, utilizamos el programa informático SPSS 22.0, realizando análisis descriptivos e inferenciales.

\section{3.- RESULTADOS}

\section{Tipo de competidor}

Sobre el tipo de raider, los competidores federados o pertenecientes a un club, practican deporte de orientación con el $93.1 \%$ y no lo practican el $6.9 \%$. Respecto a otros tipos de raiders, practican deporte de orientación con el $70.6 \%$ y no practican este deporte el $29.4 \%$ Al aplicar la estadística inferencial obtenemos, que la variable de hábito deportivo practica deporte de orientación 
influye en el tipo de raider, siendo altamente significativo con Chi-cuadrado de Pearson igual a $.000,1$ casilla (16.7\%) tiene una frecuencia esperada inferior a 5 y la frecuencia mínima esperada es 4.40.

Tabla 1. Tabla de contingencia de las variables practica deporte de aventura de orientación y tipo de raider.

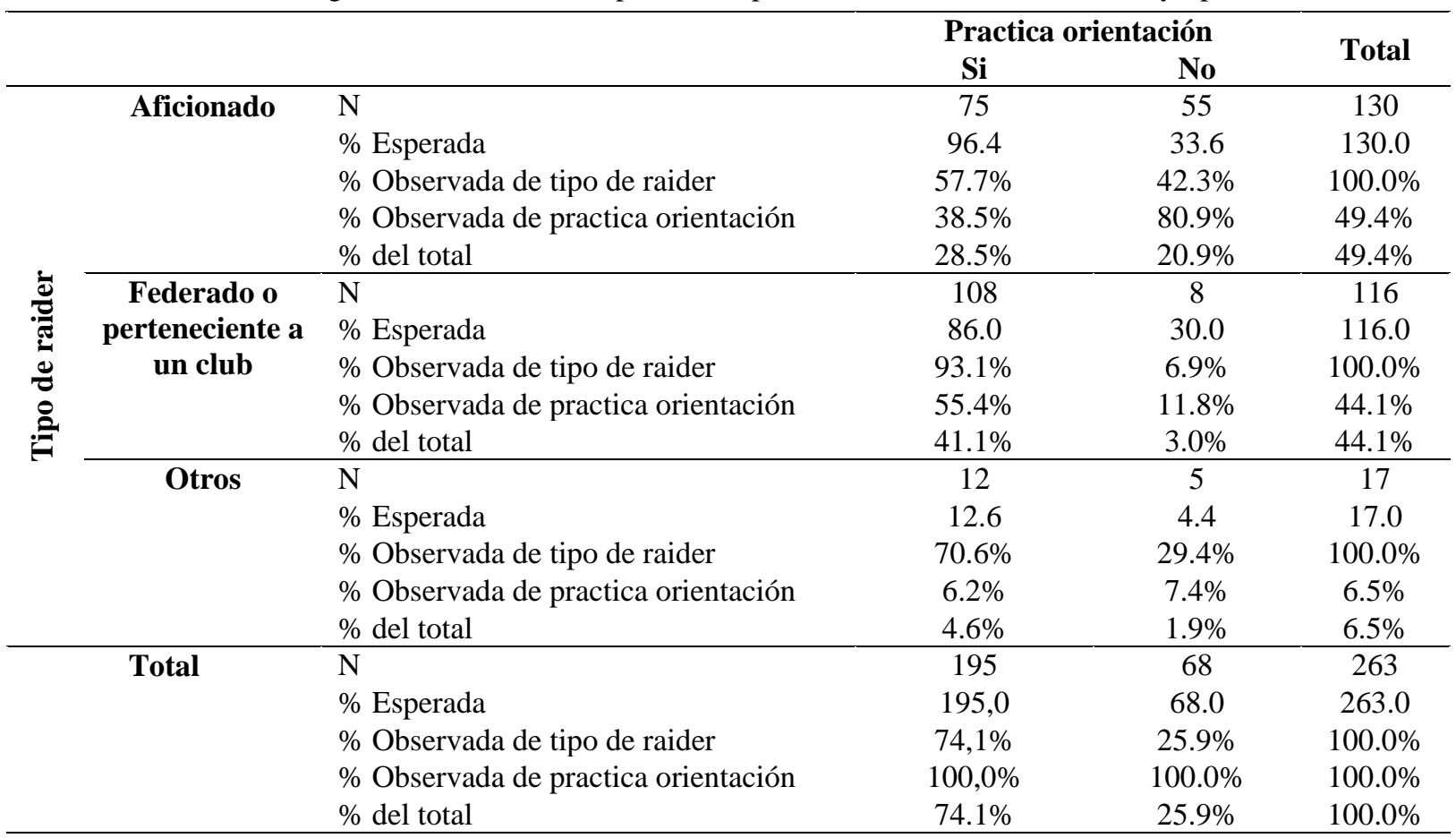

Nota. Chi-cuadrado de Pearson: Valor 40.217/GL2/Significación .000. 1 casilla (16.7\%) tiene una frecuencia esperada inferior a 5. La frecuencia mínima esperada es 4.40.

Los porcentajes de la estadística revelan que los raiders que practican orientación, son aficionados el $38.5 \%$, federados el $55.4 \%$ y otros el $6.2 \%$. Los que no practican orientación, son aficionados el $80.9 \%$, federados el $11.8 \%$ y otros el $7.4 \%$ (Figura 1 ).

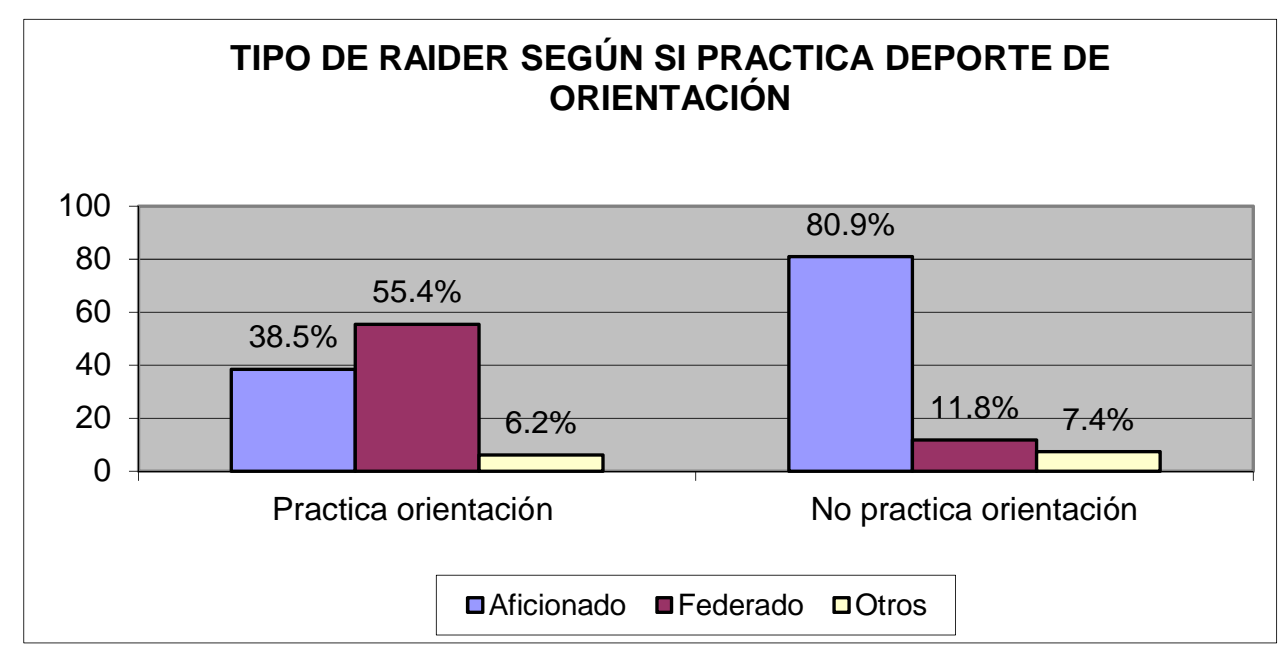

Figura 1. Distribución de frecuencias de tipo de raider según si practica deporte de aventura de orientación

\section{Mejor puesto en los últimos cinco años}

En el estudios de las frecuencias de la Tabla 2, merece la pena destacar que los campeones internacionales practican orientación con el $100.0 \%$ y no lo hacen con el $0.0 \%$ de 
los casos. Los campeones nacionales, practican este deporte con el 91.7\% y no lo practican el 8.3\% de los casos. En relación a los campeones autonómicos, practican este deporte el $97.6 \%$ de los raiders frente al $2.4 \%$ que no lo hacen. Por último, destacar que los que han conseguido otro mejor puesto en sus últimos 5 años, practican deporte de orientación con el $67.0 \%$ de los casos frente al 33.0\% que no lo practican.

Al aplicar la estadística inferencial obtenemos, que la variable de hábito deportivo practica deporte de aventura de orientación y mejor puesto en los últimos 5 años, siendo altamente significativo con Chi-cuadrado de Pearson igual a .000, 1 casilla (12.5\%) tiene una frecuencia esperada inferior a 5 y la frecuencia mínima esperada es 3.14.

Tabla 2. Tabla de contingencia de las variables practica deporte de aventura de orientación y mejor puesto en los últimos cinco años.

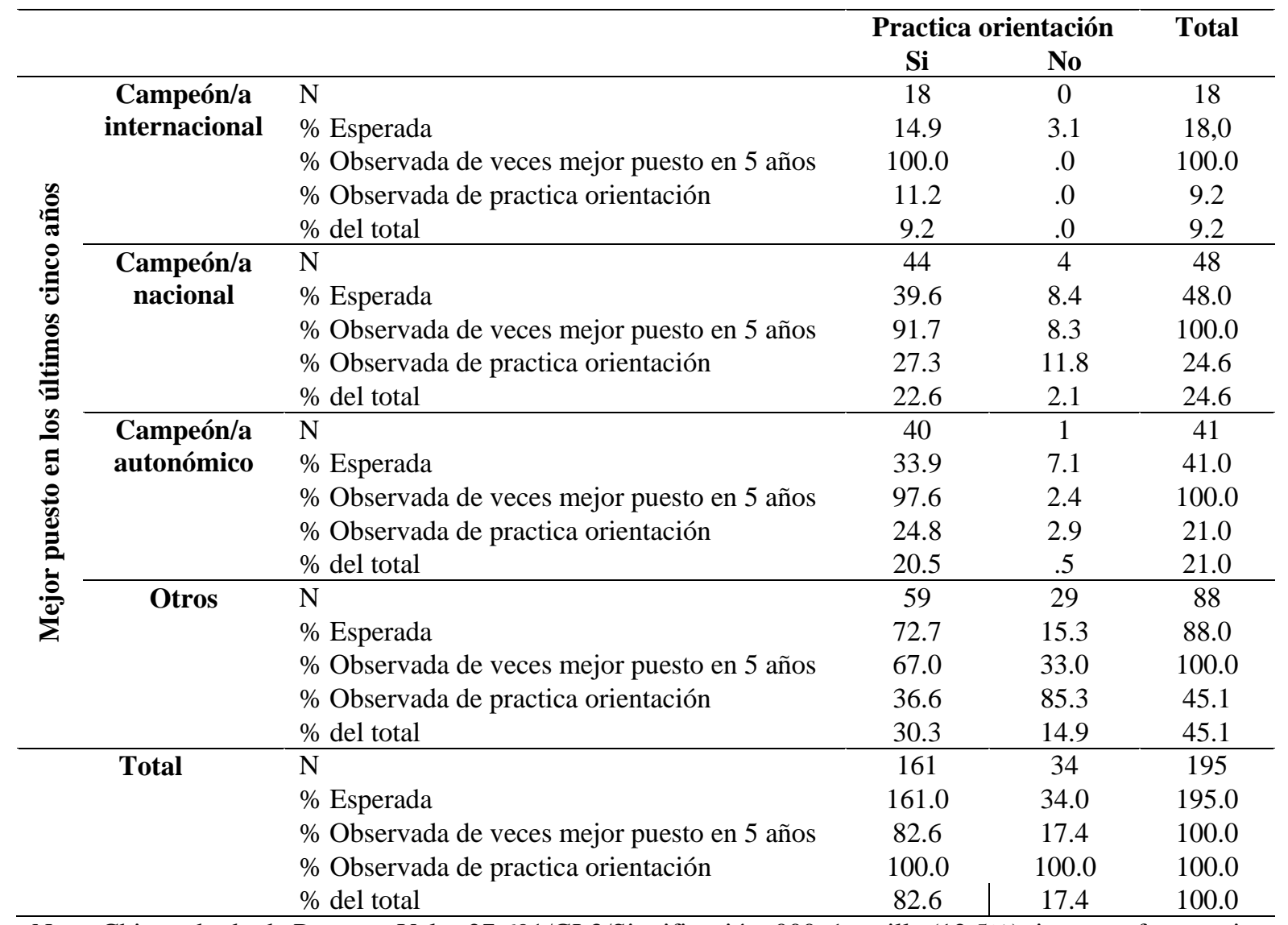

Nota. Chi-cuadrado de Pearson: Valor 27.691/GL3/Significación .000. 1 casilla (12.5\%) tiene una frecuencia esperada inferior a 5. La frecuencia mínima esperada es 3.14.

Si analizamos los porcentajes obtenidos en la estadística inferencial, observamos en la Figura 2 los siguientes resultados: los que practican deporte de aventura de orientación, el 11.2\% para campeón/a internacional, el 27.3\% campeón/a nacional, 24.8\% para campeón/a autonómico y el $36.6 \%$ para otros casos. Los que no practican deporte de aventura de orientación, el .0\% para campeón/a internacional, el 11.8\% para los campeones/as nacionales, 2.9\% para campeón/a autonómico y el 85.3\% para otros casos. 


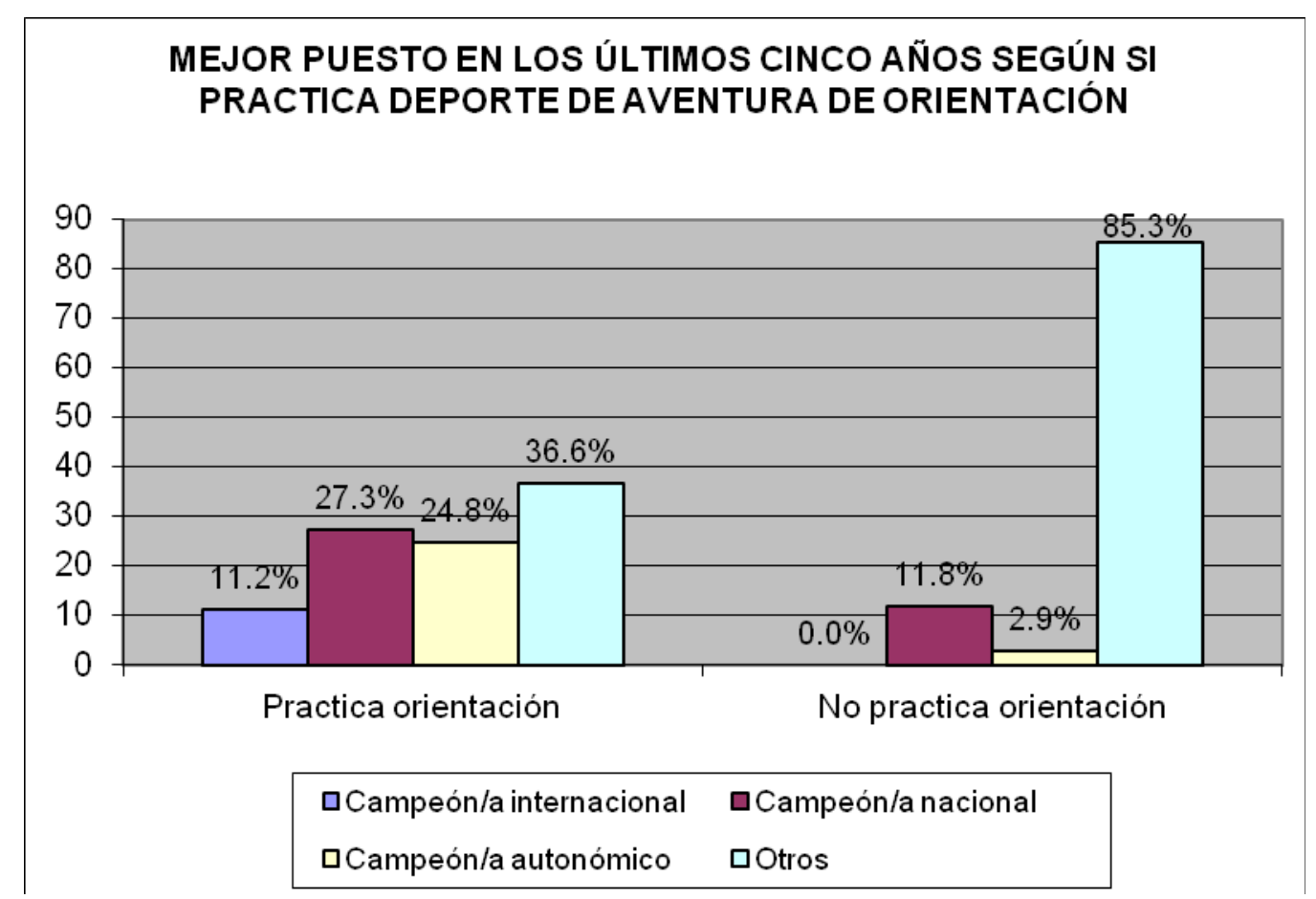

Figura 2. Distribución de frecuencias de mejor puesto en los últimos cinco años según si practica deporte de aventura de orientación.

\section{3.- CONCLUSIONES}

Entre las conclusiones de este trabajo, podemos destacar que los raider que practican orientación, son por lo general federados en deporte de aventura, pudiendo ser la orientación, lo cual, es importante, pues en los raids de aventura, uno de los deportes clave para estas pruebas es la orientación.

Pero los datos de mayor interés se muestran en el nivel conseguido por los competidores. Se observa claramente como los practicantes de orientación, son campeones en su mayoría $(11.2+27.3+24.8=52.1 \%)$ en algún tipo de prueba, nacional, autonómica o internacional, siendo el 36.6\% campeón en otro tipo de pruebas. En cambio los que no practican orientación, son en su mayoría, campeones en los últimos 5 años en otro tipo de pruebas. Igualmente, como destaca que el corredor que compite y por ello está federado, por lo general, práctica orientación, por lo que necesita formación en este deporte. De esta forma queda demostrado que para ser campeón en pruebas internacionales, autonómica y por supuesto nacionales, es necesario practicar orientación, y para ello, los futuros profesionales de este sector, tienen un gran papel.

\section{4.- REFERENCIAS}

Baena-Extremera, A. y Granero-Gallegos, A. (2011). Propuesta didáctica para el trabajo de la orientación deportiva en los centros educativos. Trances, 3(6),735-750.

Baena-Extremera, A. y Rebollo, S. (2006). Perfil Sociodemográfico y deportivo de los raiders de aventura que participan en competiciones de aventura que tienen como marco de desarrollo el ámbito nacional. IV Congreso Agesport Andalucía. Recuperado de: http://www.agesport.org/agesport/wpcontent/uploads/2009/11/comunicacion2006-02.pdf.

Baena-Extremera, A. y Rebollo, S. (2009). Análisis del perfil sociodemográfico y competitivo del practicante de raids de aventura de ámbito nacional. Apunts, Educación Física y Deportes, 98, 68-77.

Casado, J. M. (2010). El deporte de orientación en el ámbito educativo: posibilidades didácticas del gimnasio 1. EmásF: revista digital de educación física, 7, 82-94. 
Gómez, J. (2003). El uso del GPS en actividades desarrolladas en la naturaleza. Apunts, Educación Física y Deportes, 73, 70-77.

Hellín, P. y Giménez, L. (1995). Iniciación a las carreras de orientación en la naturaleza (familiarización en el medio urbano). Apunts, Educación Física y Deportes, 42, 29-35.

Kim, S. H. (2010). An exploration of the applicability of a recreational Orientierungslauf (orienteering) programme for urban cultural experience activities. Journal of Hospitality, Leisure, Sport and Tourism Education, 9(2), 130 - 140.

López, J. (2012). Propuesta de aplicación del GPS en la actividad de esquí escolar. EmasF, Revista Digital de Educación Física, 19,4.

Real Decreto 1513/2006 de 7 de diciembre por el que se establecen las enseñanzas mínimas de la Educación Primaria. (B.O.E., $\mathrm{n}^{\circ} 293$ del 8 de diciembre).

Real Decreto 1631/2006 de 29 de diciembre, por el que se establecen las enseñanzas mínimas de la Educación Secundaria Obligatoria (B.O.E., n ${ }^{\circ} 5$ de 5 de enero).

Schneider, T. A., Butryn, T. M., Furst, D. M., Masucci, y M. A. (2007). A qualitative examination of risk among elite adventure racers. Journal of Sport Behavior, 30, 3- 330.

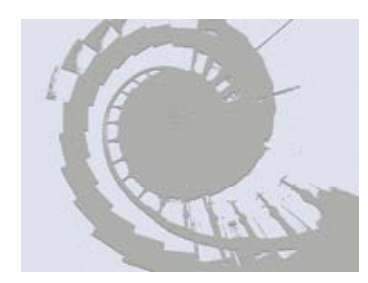

